\title{
Ruptured popliteal cyst simulating deep vein thrombosis with false positive radiofibrinogen uptake
}

\author{
D. GiBBONS \\ M.B., M.R.C.P.
}

\author{
M. PHILliPS
}

B.Sc., M.R.C.P.

\author{
IRENE M. Prossor \\ M.B., B.S., F.F.R.
}

The Gordon Hospital and Westminster Hospital, London SW.1

\begin{abstract}
Summary
A case is described in which deep vein thrombosis of the calf was initially diagnosed on the basis of the clinical signs and the radiofibrinogen uptake in a patient suffering from rheumatoid arthritis. The correct diagnosis of ruptured popliteal cyst was later established by arthrography and phlebography. Attention is drawn to the unreliability of the radiofibrinogen uptake test in the presence of ruptured popliteal cyst and alternative diagnostic methods are discussed.
\end{abstract}

\section{Introduction}

Ruptured popliteal (Baker's) cysts may present with symptoms and signs closely mimicking those of deep vein thrombosis of the calf (Hooper and Brookler, 1971). In order to differentiate between these two conditions the uptake of ${ }^{125}$ I-labelled fibrinogen would appear to be a suitable test since it can be a sensitive indicator of deep vein thrombosis with a low incidence of false positive results (Browse et al., 1971). However, a case is described in which a patient whose radiofibrinogen uptake test was positive, was initially diagnosed as suffering from deep vein thrombosis of the calf. He was later shown by arthrography and phlebography to have a ruptured cyst and no evidence of venous thrombosis.

\section{Case report}

A 54-year-old laboratory technician was first seen in May 1973 with rheumatoid arthritis of acute onset (titre for rheumatoid factor $1: 512$ ). The joints mainly involved were those of the hands and feet, knees, right ankle and left shoulder. He was treated with a variety of anti-inflammatory agents (but not with corticosteroids). In November 1973 he consulted his general practitioner because his left knee had again become swollen and painful with the development of a painless lump behind. This was diagnosed as a Baker's cyst. To control the swelling he used a tight elastic bandage and 5 days later the swollen knee had abated and the lump behind disappeared. At this time the left calf became swollen and felt tense and he was admitted to hospital with a diagnosis of deep vein thrombosis.

On examination there was moderate oedema around the left ankle and lower two thirds of the leg. The calf was warm and tender but Homan's sign was negative. Midcalf circumference was $36 \mathrm{~cm}$ on the left and $33 \mathrm{~cm}$ on the right. Effusions were present in both knee joints.

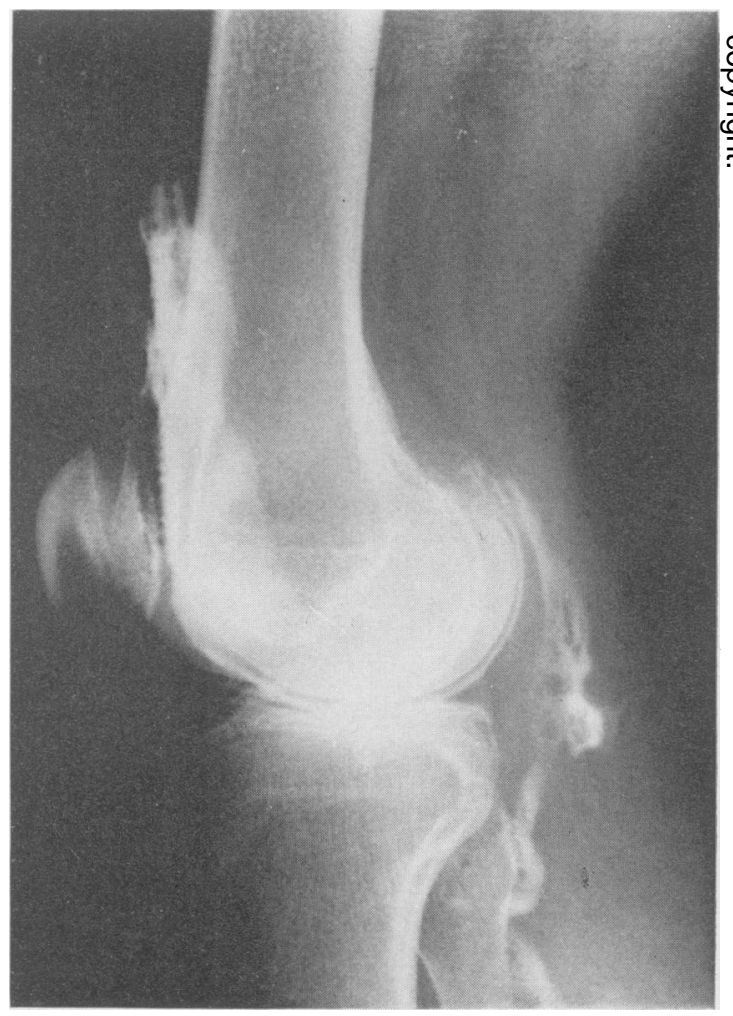

Fig. 1. Arthrogram (lateral view) with contrast and air tracking into the calf from a posterior cyst communicating with the joint. 
The radioactive fibrinogen scan was greater than five times normal over a line extending down the surface projection of the popliteal vein, which was taken as unequivocal evidence of a deep vein thrombosis of the calf.

He was treated with a continuous infusion by syringe pump of heparin, 20,000 units 12-hourly, and the symptoms and signs gradually abated. Nine days later he developed a haematoma of the left calf and the original diagnosis was queried. A venogram was then performed, showing that the deep veins were patent from the ankle to the inguinal ligament with a constant narrowing of the popliteal vein suggestive of external compression. A left knee arthrogram performed at the same time showed air and contrast passing down the calf, the appearances being those of a ruptured Baker's cyst (Fig. 1).

\section{Discussion}

Acute rupture of popliteal cysts, or the distended synovial joint of the knee in rheumatoid arthritis is well recognized as a condition that may resemble deep vein thrombosis (Dixon and Grant, 1964; Tait, Bach and Dixon, 1965; Hughes and Pridie, 1970; Hooper and Brookler, 1971). Failure to distinguish between the conditions may lead to anticoagulants being given in error and the development of complications. Our patient sustained a haematoma of the calf which fortunately resolved, but in one series this event was followed by muscle contracture and a prolonged and difficult convalescence (Tait et al., 1965).

On clinical grounds alone the differentiation can be difficult. In making the diagnosis of ruptured popliteal cyst the only consistently valuable sign is the presence of an ipsilateral synovitis or effusion in the knee joint (Hooper and Brookler, 1971). However, this does not exclude the possibility of a deep vein thrombosis occurring in the presence of a rheumatoid involvement of the knee.

Of the diagnostic aids capable of distinguishing between thrombophlebitis and cyst rupture, contrast arthrography of the knee has been described as the single most valuable investigation (Peri, Rodnan and Mankin, 1968). More recently ultrasound Bscanning has been shown to provide a simple and rapid non-invasive technique for differentiating between the two conditions (McDonald and Leopold, 1972).
The ${ }^{125}$ I fibrinogen uptake test has an established $\stackrel{\$}{\otimes}$ place in the diagnosis of deep vein thrombosis, $C$ although false positive results have been obtained in $\Rightarrow$ the presence of superficial phlebitis, varicose ulcera- $\stackrel{S}{?}$ tion, sepsis, flesh wounds and active arthritis. Patients with these conditions should normally be $\overline{ }$. excluded from having the test (Browse et al., 1971). $\frac{\bar{\sigma}}{\overrightarrow{0}}$

In the case described, effusions were present in $\stackrel{\varnothing}{\varrho}$ both knees, and it was thought reasonable to expect one side to act as a control. No increase in radio- $\vec{\circ}$

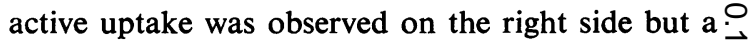
high uptake was found over the course of the left $\vec{\omega}$ popliteal vein and this was erroneously interpreted as 9 indicating a deep vein thrombosis.

Following synovial rupture fluid and blood track into the muscles of the calf and can be aspirated from 0 this site (Tait et al., 1965). It is therefore in retro- 8 spect not surprising that the ${ }^{125}$ I-uptake test was 0 positive. This emphasizes that all acute swellings of $\omega$ the calf in patients with known effusions of the knee $\mathrm{G}$ should be considered as cases of acute synovial 은 rupture until proved otherwise (Tait et al. 1965) and $\vec{\circ}$ that the ${ }^{125}$ I fibrinogen test cannot be relied upon to exclude a deep vein thrombosis.

\section{Acknowledgments}

We would like to thank Dr A. F. Lant (Senior Lecturer Therapeutics and Consultant Physician, Westminist Hospital) for permission to report this case.

\section{References}

Browse, N.L., Clapham, W.F., Croft, D.N., Jones, D.J. \& $\stackrel{\mathbb{D}}{\varrho}$ Thomas, M.L. (1971) Diagnosis of established deep vein thrombosis with the ${ }^{125}$ I fibrinogen uptake test. 윽 British Medical Journal, 4, 325.

Dixon, A. ST. J. \& GRANT, C. (1964) Acute synovial rupture in rheumatoid arthritis. Lancet, $\mathbf{i}, 742$.

Hooper, J.C. \& Brookler, M. (1971) Popliteal cysts and their rupture in the rheumatoid arthritic simulating thrombophlebitis. Medical Journal of Australia, 1, 1371.

Hughes, G.R. \& Pridie, R.B. (1970) Acute synovial rupture of the knee a differential diagnosis from deep vein thrombosis. Proceedings of the Royal Society of Medicine, 63, ᄋ 587.

McDonald, D.G. \& Leopold, G.R. (1972) Ultrasound B-scanning in the differentiation of Baker's cyst and $\stackrel{9}{\supset}$ thrombophlebitis. British Journal of Radiology, 45, 729.

Peri, J.A., Rodnan, G.P. \& MankIN, H.J. (1968) Giant 을. synovial cysts of the calf in patients with rheumatoid arthritis. Journal of Bone and Joint Surgery, 50A, 709.

TAIT, G.B.W., BACH, F. \& Dixon, A. ST. J. (1965) Acute synovial rupture. Annals of Rheumatic Diseases, 25, 273. 